Article

\title{
Pre- and Post-Launch Spatial Quality of the Landsat 8 Thermal Infrared Sensor
}

\author{
Brian N. Wenny ${ }^{1, *}$, Dennis Helder ${ }^{2}$, Jungseok Hong ${ }^{2}$, Larry Leigh ${ }^{2}$, Kurtis J. Thome ${ }^{3}$ \\ and Dennis Reuter ${ }^{3}$
}

1 Sigma Space Corporation, 4600 Forbes Blvd., Lanham, MD 20706, USA

2 Office of Engineering Research, College of Engineering, South Dakota State University, Brookings, SD 57007, USA; E-Mails: dennis.helder@sdstate.edu (D.H.); jungseok.hong@sdstate.edu (J.H.); larry.leigh@sdstate.edu (L.L.)

3 NASA Goddard Space Flight Center, Greenbelt, MD 20771, USA;

E-Mail: kurtis.thome@nasa.gov (K.T.); dennis.c.reuter@nasa.gov (D.R.)

* Author to whom correspondence should be addressed; E-Mail: brian.n.wenny@nasa.gov; Tel.: +301-876-2087

Academic Editors: Ron Morfitt and Prasad S. Thenkabail

Received: 8 August 2014 / Accepted: 28 January 2015 / Published: 11 February 2015

\begin{abstract}
The Thermal Infrared Sensor (TIRS) for the Landsat 8 platform was designed and built at NASA Goddard Space Flight Center (GSFC). TIRS data will extend the data record for thermal observations from the heritage Landsat sensors, dating back to the launch of Landsat 4 in 1982. The two-band (10.9 and $12.0 \mu \mathrm{m})$ pushbroom sensor with a $185 \mathrm{~km}$-wide swath uses a staggered arrangement of quantum well infrared photodetector (QWIPs) arrays. The required spatial resolution is $100 \mathrm{~m}$ for TIRS, with the assessment of crop moisture and water resources being science drivers for that resolution. The evaluation of spatial resolution typically relies on a straight knife-edge technique to determine the spatial edge response of a detector system, and such an approach was implemented for TIRS. Flexibility in the ground calibration equipment used for TIRS thermal-vacuum chamber testing also made possible an alternate strategy that implemented a circular target moved in precise sub-pixel increments across the detectors to derive the edge response. On-orbit, coastline targets were developed to evaluate the spatial response performance. Multiple targets were identified that produced similar results to one another. Even though there may be a slight bias in the point spread function (PSF)/modulation transfer function (MTF) estimates towards poorer performance using this approach, it does have the ability to track relative changes for
\end{abstract}


monitoring long-term instrument status. The results for both pre- and post-launch response analysis show general good agreement and consistency with edge slope along-track values of 0.53 and 0.58 pre- and post-launch and across-track values 0 f 0.59 and 0.55 pre- and post-launch.

Keywords: Landsat; TIRS; spatial calibration

\section{Introduction}

Landsat 8 was launched on 11 February 2013, with a mission to continue into a fifth decade the measurements of the heritage Landsat sensors. This dataset is critical for the understanding of land use changes and resource management [1]. Landsat 8 is a two-sensor platform comprised of the Operational Land Imager (OLI) and the Thermal Infrared Sensor (TIRS), with OLI operating in concert with, but independent of, TIRS. Both instruments are push-broom sensors with a roughly $185 \mathrm{~km}$-wide swath. The OLI instrument collects data at visible, near-infrared and shortwave infrared wavelengths, whereas the TIRS instrument collects longwave infrared wavelength data. The wavelengths are chosen to match those of the previous Landsat sensors. TIRS is a two-band sensor (10.9 and $12.0 \mu \mathrm{m})$ with $100-\mathrm{m}$ spatial resolution that was built by NASA Goddard Space Flight Center.

The TIRS optical system consists of a four-element refractive telescope directing the incoming beam onto the focal plane. A flat mirror, known as the scene select mechanism (SSM), is attached in front of the telescope and can rotate through three set positions: nadir, a space view port and the on-board temperature-controlled calibration blackbody. The telescope optics are passively cooled at an operational temperature of approximately $186 \mathrm{~K}$. A mechanical cryocooler actively controls the focal plane to temperatures below $40 \mathrm{~K}$. The focal plane is made up of three quantum well infrared photodetector arrays (QWIP), each containing 512 by 640 detectors. Interference filters installed on the focal planes provide the spectral selection for the two bands. Three QWIP array sensor-chip assemblies (SCA A, B and C) are needed to cover the entire swath of TIRS. Each SCA is a fully populated 2D array, over which the spectral filters for each band are placed along with an aluminum mask to provide a "dark" measurement. Two rows of detectors (from a possible 32 rows) are selected from each band, as well as two rows from under the mask, to give a total of six rows of data compiled to generate a TIRS image. The on-board calibrator blackbody is a curved-plate with a V-groove design to improve the overall emissivity. The design and coating are very similar to that used for MODIS to give high emissivity and controllable temperature. The output from the blackbody is NIST traceable and capable of providing sources of varying temperature. Detailed descriptions of the TIRS instrument are available in [2,3].

The Landsat program has been a success, in large part because of an emphasis on the knowledge of the calibration of the sensors, both geometric and radiometric [4]. The radiometric and geometric assessment of the sensors has relied on a combination of both pre-launch and post-launch efforts using a variety of sources and methods. TIRS pre-launch calibration and characterization was completed successfully at NASA GSFC using a custom-built calibration ground support equipment (CGSE) system. Since commencing routine operations in March, 2013, TIRS has demonstrated excellent performance [5]. This paper addresses the spatial characterization assessment of the TIRS instrument, both pre- and post-launch. A brief description of sensor spatial quality follows. Section 2 describes the methods and 
approaches for determining the spatial quality. Section 3 details the methodologies used both pre-launch and post-launch. The results obtained from the two mission phases are discussed in Section 4, followed by conclusions.

\section{Sensor Spatial Performance}

The spatial quality of an optical remote sensing satellite image is often a difficult concept to define, as it involves many aspects of the imaging system. However, in general, spatial quality refers primarily to the interaction between ground sample distance (GSD) and the point spread function (PSF) of an imaging system. The GSD is the distance between centers of adjacent pixels and is given nominally in the horizontal and vertical directions. The PSF of an imaging system is a measure of the amount of blurring that occurs due to all of the components that comprise the imaging system. All of the energy received at the detector of the imaging system is not uniformly distributed across the area of the detector as transferred from the projected pixel on the surface and, in fact, always includes energy coming from outside the nominal instantaneous field of view for a given detector. Thus, there is a complex interaction among all components of the imaging system (optics, detectors, electronics and atmosphere) forming the PSF and, ultimately, the spatial quality of the system. A more detailed review of this relationship can be found in $[6,7]$.

Spatial quality is often described in terms of the PSF. However, the PSF is a two-dimensional function that is easier to predict based on theory than to measure prior to launch or on-orbit. To overcome this limitation, other simpler metrics are often used. The edge spread function (ESF) is the response of the system to an edge that is often nominally oriented in the horizontal or vertical direction and can be thought of as essentially a one-dimensional integration of the PSF. Per the TIRS requirements, the edge slope is the rate of increase in intensity from the 0.4 to 0.6 amplitude levels of the ESF. The edge slope has seen an increase in popularity for specifying the performance of satellite imaging systems, with Landsat 8 being a recent example. Conversely, the modulation transfer function (MTF) has historically been a widely-used figure of merit with the value of the MTF at the Nyquist frequency, often forming the system design requirement for sensors. An excellent overview of these measures and their development can be found in [8]. Because ESF and the line spread function (LSF) are often more intuitive than MTF, the results developed in this paper will focus largely on the ESF measurement and its derivative, LSF, as an assessment of the spatial quality of the TIRS instrument.

Pre-launch characterization of any remote sensing instrument will include an assessment of sensor spatial quality. The typical approach to collecting the needed measurements is the use of an illuminated "knife-edge" target. The edge in this case is between full illumination and a dark background. This type of target provides all of the information needed to analyze and derive spatial performance parameters, such as edge slope, ESF and MTF. This approach was used for TIRS pre-launch characterization. The spatial analysis of pre-launch data was focused on addressing the instrument requirements for spatial quality, which were expressed in terms of edge slope and edge extent. The edge extent is the ground distance between the 0.1 and 0.9 amplitude levels of the ESF. Determining spatial quality once on orbit is more challenging; thus, careful pre-launch characterization is imperative.

On-orbit estimation of MTF for thermal imaging satellite sensors has not often been performed. This is due, at least partially, to the difficulty in obtaining a sharp edge target for thermal wavelengths, as 
well as because of the large IFOV typical of these sensors. Examples of efforts for large IFOV sensors include PSF/MTF estimation for MODIS [9] and GOCI [10]. However, because of the legacy of thermal sensors with the Landsat series of satellites dating back to 1984 with the Landsat 4 Thematic Mapper and with the recent launch of the Landsat 8 TIRS, an effort was undertaken to establish a methodology to measure at least the stability of the PSF/MTF of these sensors and, also, if possible, to accurately estimate the PSF/MTF itself.

Many approaches have been developed for measuring the spatial response for optical sensors on orbit. For the most part, these can be classified by the type of target that is being used: edge targets, point targets, line targets and pulse targets $[8,11]$. Although not used universally, edge targets are probably the most widely utilized type of on-orbit targets, as both man-made and natural objects can be identified as suitable edge targets.

Edge targets are normally aligned with the orbital velocity vector of the satellite, such that the edge response function can be obtained in either the along-track or cross-track direction. The alignment is typically rotated a few degrees from these directions, so that it is not perfectly aligned, and the edge may be sampled in a manner to allow sub-pixel reconstruction, as shown in the lower half of Figure 1. Each row of pixel information is properly aligned to form an over-sampled edge, as shown in the upper half of Figure 1. Following numerical differentiation to obtain the one-dimensional point spread function, commonly called the line spread function (LSF), estimations of full-width half-maximum (FWHM) and relative edge response can be obtained. Details on how these calculations are performed can be found in [12].

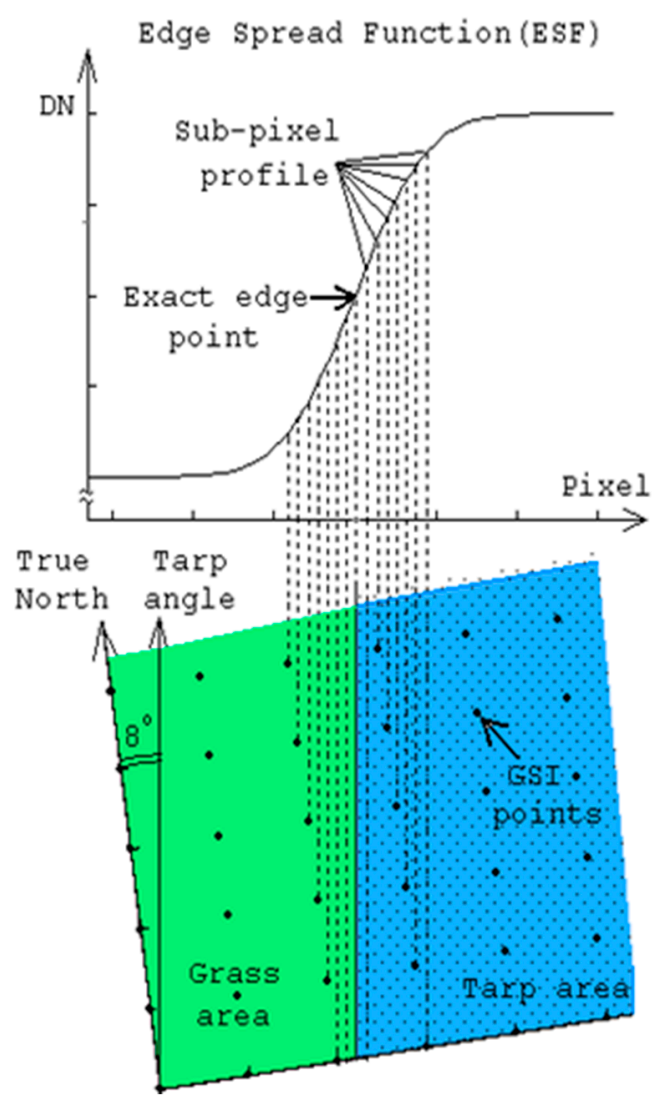

Figure 1. Sampling of an edge target by a satellite sensor emphasizing the orientation of the edge with respect to the satellite sampling grid (bottom). The figure shows how the orientation of the edge leads to a well-sampled edge spread function (top). 
Point targets are very attractive to use, because they can provide an estimate of the full two-dimensional PSF. However, difficulties with these targets include low intensity levels, leading to low SNR (stars are a good example), the possible need to point the targets accurately to track the sensor's field of view and the need for large numbers of targets to obtain adequate sampling of the 2D PSF. Mirrors have been successfully deployed to obtain these estimates [11], as have spotlights [13]. For those satellite sensors that are pointable, stars have been used to assess the sensor spatial response [14].

An additional type of target is called the pulse target and is based on the use of a uniform width bright area on a dark background. This type of target is often used with those sensors that have an IFOV larger than a few meters, because edge targets are hard to develop for this scale of spatial resolution. Instead, a "narrow pulse" is used in a region with a relatively uniform background. Using this approach, a parameterized model can be fit to the pulse response function in the imagery if an underlying model may be assumed for the sensor PSF [15]. In the case where an underlying model is not known, a non-parametric approach may be taken in the Fourier domain, where the input is a known pulse with a sinc function as the Fourier transform. The pulse response, as estimated from the imagery, can then be Fourier-transformed and ratioed to the input sinc function to obtain the sensor MTF [12].

\section{Methodology}

\subsection{Pre-Launch}

The TIRS pre-launch calibration and characterization was conducted at NASA GSFC facilities. The instrument was placed in a thermal vacuum (TVAC) chamber to simulate the expected on-orbit operational environment. The custom-built CGSE system contained an ensemble of optics, baffles and electromechanical devices affixed to a common mounting plate and was designed to provide TIRS with an infrared source for calibration and characterization. The CGSE IR source used for the spatial characterization test was the infrared source module (IRSM) with a NIST traceable blackbody and a motorized filter wheel containing numerous target-aperture shapes. Additionally, the IRSM contained linear stages, which, combined with a steering mirror, allowed precise, repeatable positioning of any selected target onto the TIRS FPA. Details of the CGSE system are available in [16]. During spatial characterization testing, the IRSM blackbody temperature was controlled at $300 \mathrm{~K}$. The target shapes used in spatial testing were a blank for background, a large square $(90 \times 90$ pixels $)$ and a 16-pixel diameter circle. The square and circle targets have the same center location. An example image of the circular target is shown in Figure 2. The individual rows/columns of pixels can be seen in the image, as well as the drop off in signal evident at the edges of the circular target image. Initially, the large square target was used for edge analysis; however, preliminary testing indicated a vignetting issue near the edges of the square target, so that necessitated a change to develop an analysis strategy using the circular target.

The test measurement procedure consisted of positioning the circular target on a region on the FPA and then collecting a sequence of images using the blank, square and circular targets. All image frames collected were of the full $32 \times 640$ active pixel regions of each band and SCA. The CGSE steering mirror allowed movement of the image in sub-pixel incremental steps (1/5 pixel). The measurement sequence included multiple image frames recorded while the target incremented in cross-track and along-track 
directions. The collection sequence was repeated at locations on all three active SCA arrays and bands to sample across the full FOV. To derive the edge response, each dataset (for any particular location) was processed as follows. The background correction was determined using an average of the collected blank target frames. Each frame of the circular and square targets was background corrected by subtracting the average background image. To flat field the circular target images, which removes any pixel-to-pixel response variations, each image frame of the background-corrected circular target was divided by the average of all background-corrected square target frames. Figure 3 shows all across-track (horizontal) cross-sections of a typical background-corrected, flat-fielded circle image (such as the one seen in Figure 2). The maximum horizontal cross-section was determined for each image, indicated as the black curve in Figure 3. Each frame was normalized by the average digital count (dn) response of the 5 center pixels in the maximum horizontal cross-section. All 80 maximum frames collected during an incremental across-track move dataset are shown in Figure 4. It can be seen that the spread on the right and left edges of the target image is due to the series of 1/5th-pixel incremental moves. The edge response for each frame is fit to a Fermi function to derive the edge midpoint. All frames are shifted to a common pixel reference scale by matching up the edge midpoints. An example of the result of this shifting is shown in Figure 5. By combining all frames of shifted data, a more complete sampling of the image edges is obtained. The Fermi function used in this analysis is [12]:

$$
f(x)=\frac{a}{\left(e^{(x-b) / c}\right)+1}+d
$$

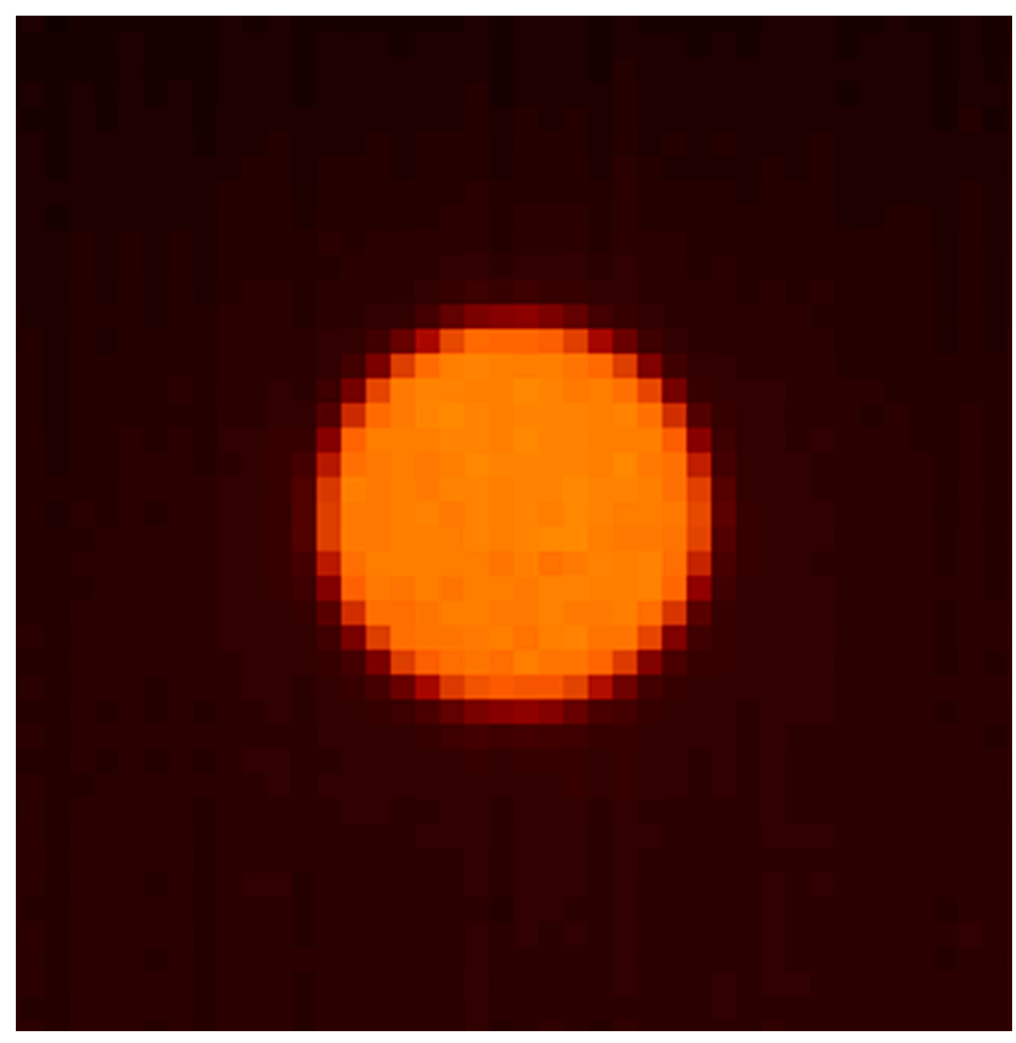

Figure 2. Raw image frame of the calibration ground support equipment (CGSE) infrared source module (IRSM) 16-pixel diameter circular target from the pre-launch testing. 


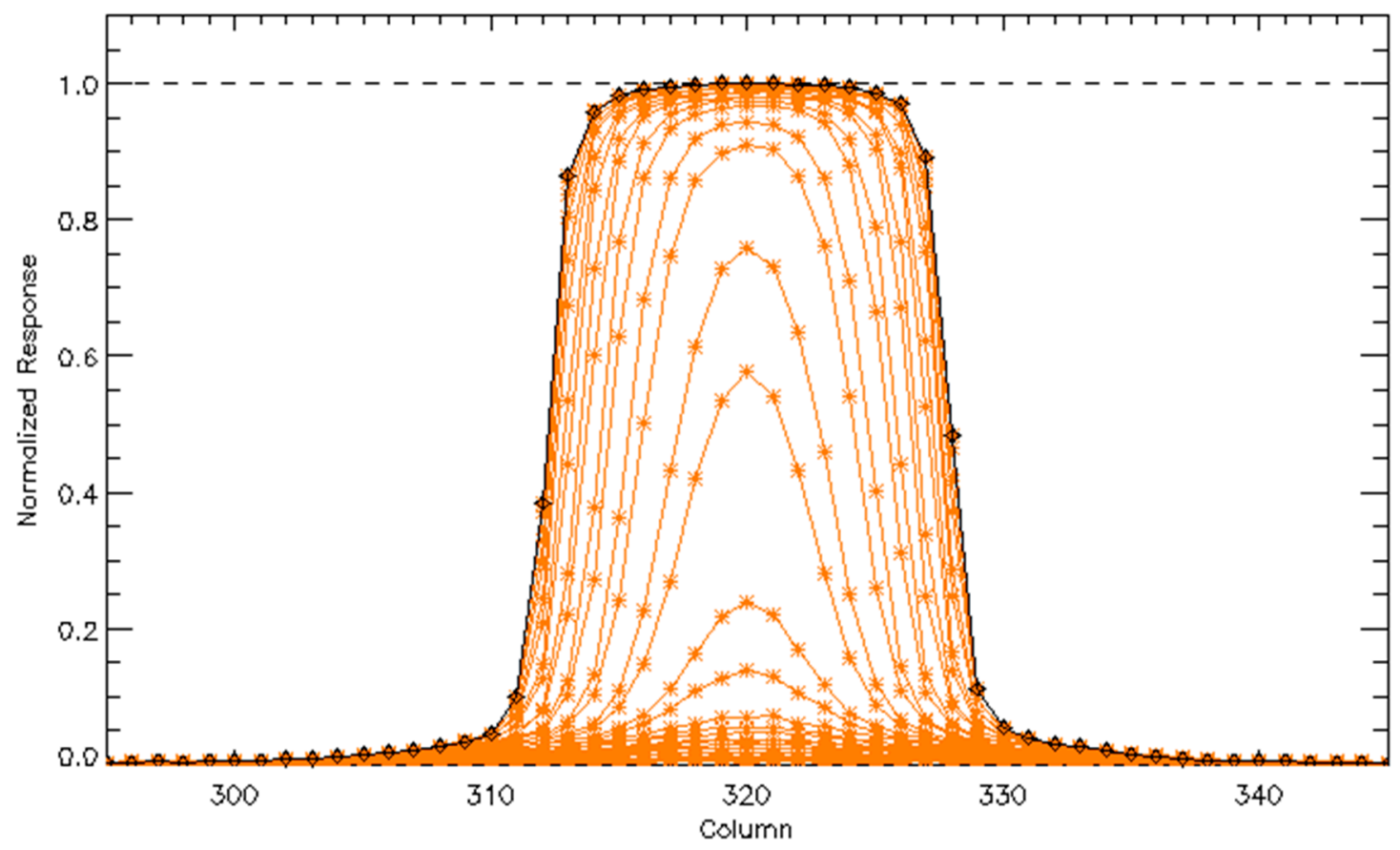

Figure 3. Peak normalized cross-track cross-sections of the circular target image for all 32 active rows in the illuminated band/sensor-chip assembly (SCA).

The shift edge response is resampled using linear interpolation to uniform pixel spacing (0.01 pixel) for the determination of the $0.4,0.6$ (for relative edge response), 0.1 and 0.9 (for edge extent) response points. The left edge of Figure 5 is shown in more detail in Figure 6 . The dashed horizontal lines in Figure 3 indicate the various response points defined by the requirements. This process was repeated for all datasets collected for both cross-track and along-track sub-pixel incremental image movement.

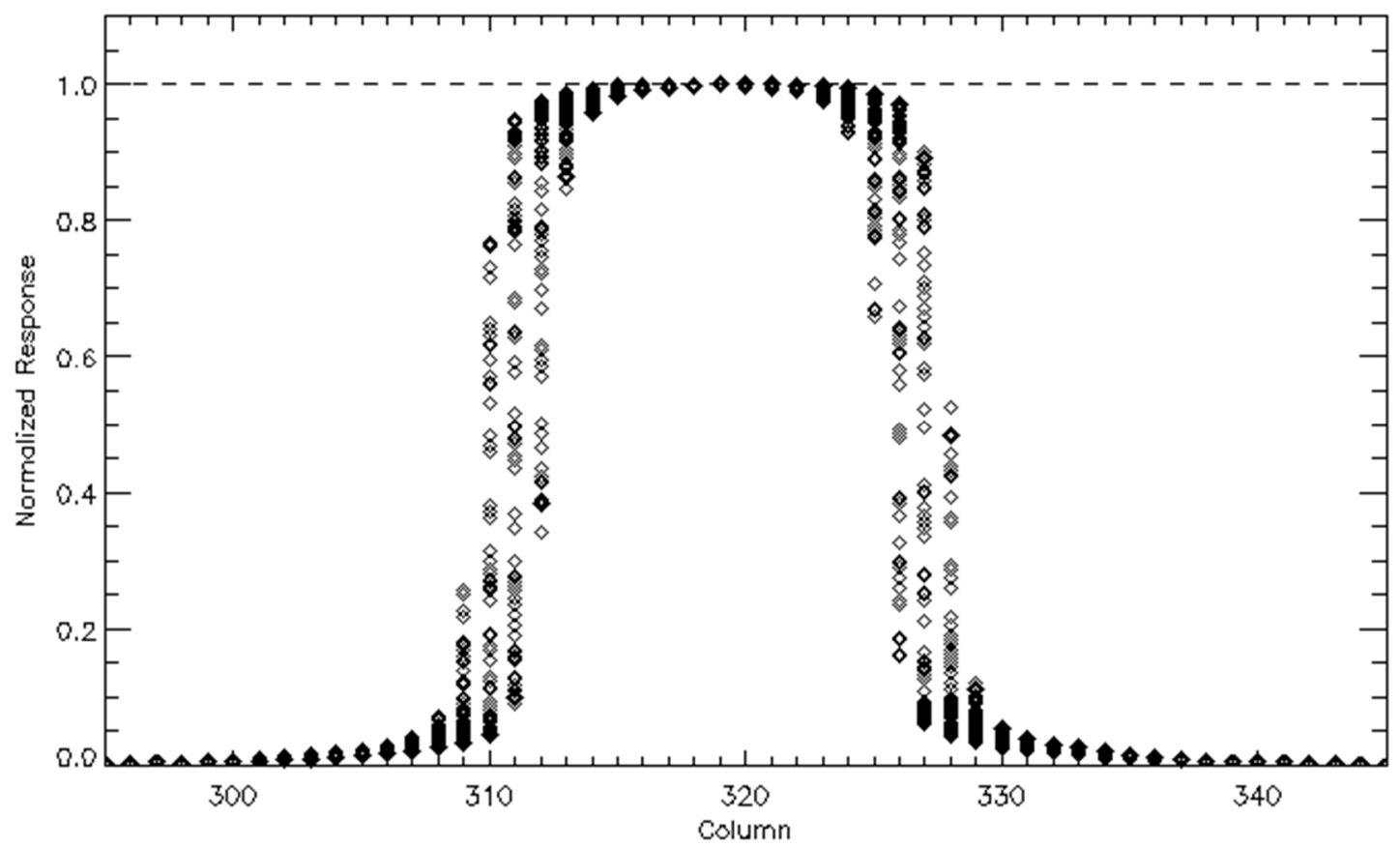

Figure 4. All 80 maximum peak normalized cross-track cross-sections collected for a sub-pixel incremental cross-track image move. 


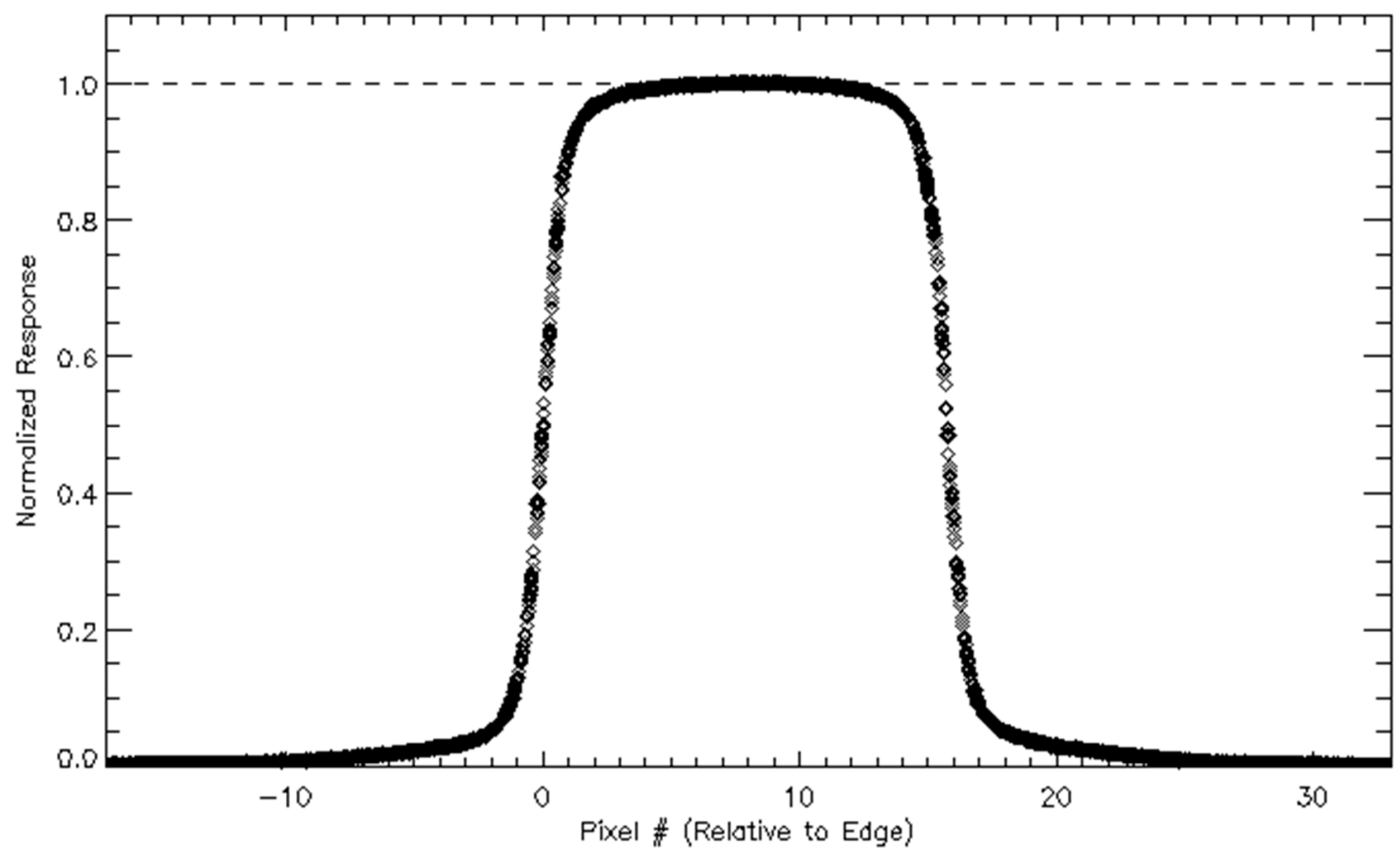

Figure 5. Cross-sections from Figure 4 shifted to a common reference frame.

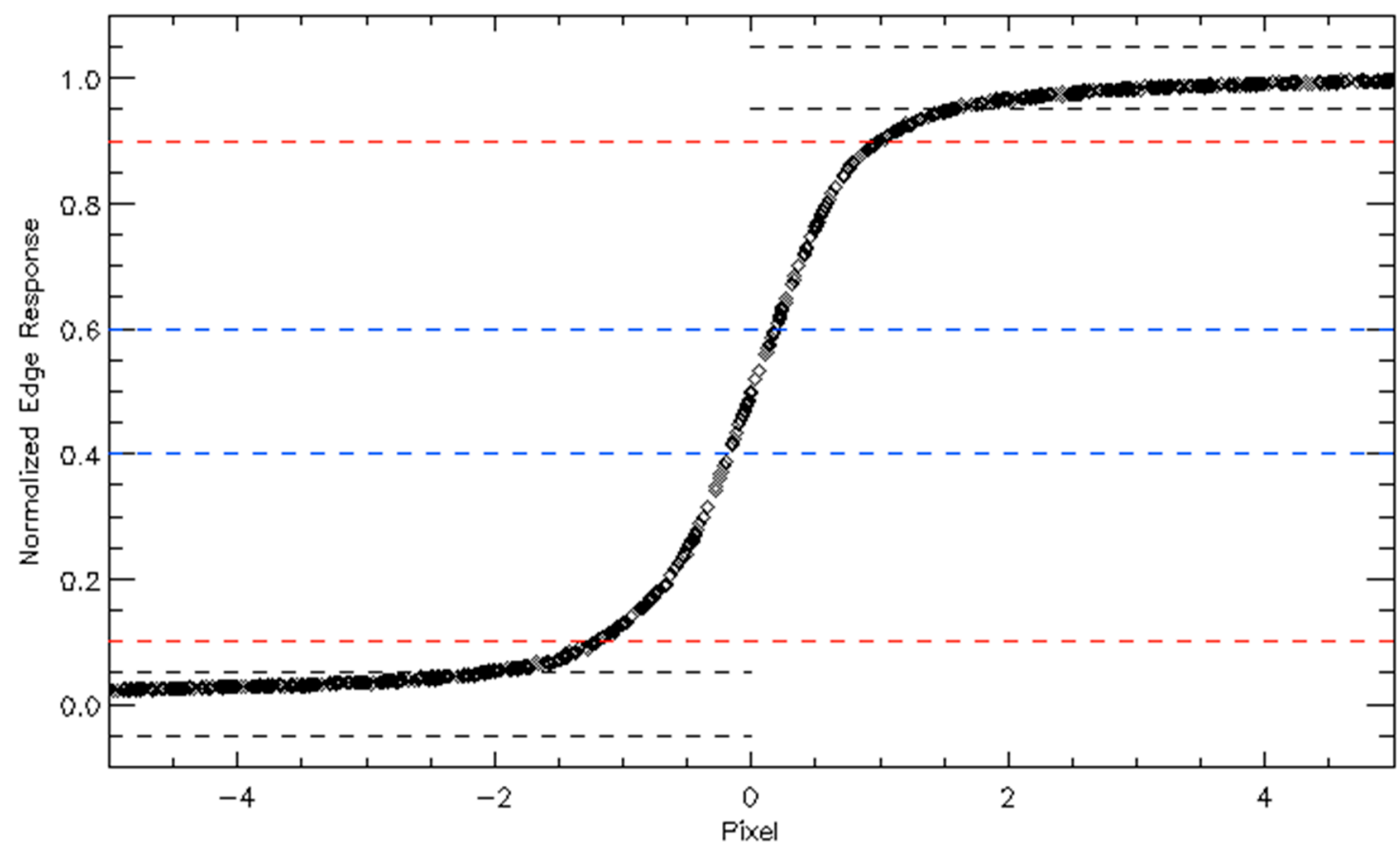

Figure 6. Left edge of Figure 5.

\subsection{Post-Launch}

Because of the large IFOV of the Landsat thermal sensors, the development of a man-made target for PSF/MTF estimation was deemed impractical, and the focus for target development quickly became centered on naturally-occurring targets. Stable, well-defined point sources do not regularly occur in nature, so some type of target with edges is necessary, i.e., a line, pulse or edge target. One of the difficulties is that naturally-occurring thermal edges are also difficult to obtain. One of the better 
possibilities, however, was to consider shorelines of colder water and warmer land. In order to optimize the target suitability, additional properties to consider are: (1) the maximal difference between the temperature of the water and land; (2) the thermal uniformity of the land; (3) the physically stable shoreline (minimal effects seasonally and with respect to tides); (4) the sharp transition between water and dry land (minimal extent of wetted surfaces); (5) the proper orientation with respect to the satellite orbit; and (6) the minimal changes in temperature of both water and land seasonally and with respect to distance from the shoreline.

\subsubsection{Site Selection}

Given the criteria listed above, a search was made for coastlines in the Middle East and African desert regions to identify candidate targets for PSF/MTF estimation of Landsat 8 TIRS. Many regions of shorelines could be quickly eliminated by the constraint on orientation. Since Landsat is in a polar orbit at an inclination of $98^{\circ}$ and a nearly optimal angle for the shoreline is $\pm 8^{\circ}$ from this orientation for cross-track estimations, large areas of shorelines could be easily removed from consideration. However, in addition to this orientation, PSF/MTF estimation in the along-track direction is best estimated with a shoreline angle of $8 \pm 8^{\circ}$, and shorelines with this orientation were also considered. High-resolution visible imagery was used to quickly identify these regions. Once candidate lists of possible targets had been formed, Landsat thermal band imagery of those locations was evaluated visually and quantitatively to determine how well the rest of the criteria were met.
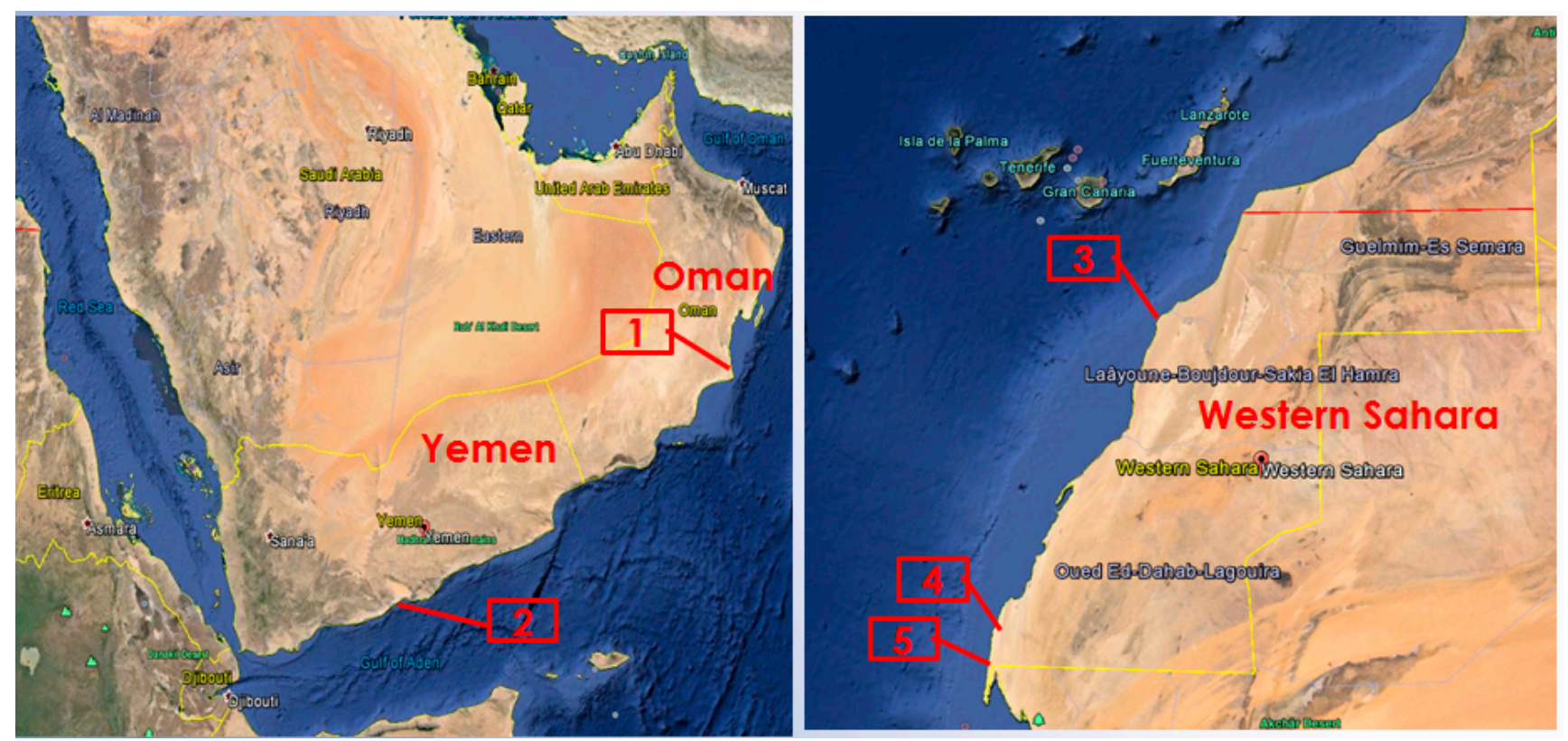

Figure 7. Location of the target sites for the estimation of Landsat 8 TIRS point spread function (PSF)/modulation transfer function (MTF).

Based on this procedure, five candidate sites were selected. Two were in the Middle East, one on the shore of Oman and one on the shore of Yemen. Three sites were selected from the shoreline of Mauritania on the northwest coast of Africa. These were named Western Sahara 1, 2 and 3 and were three different locations in the same Landsat Worldwide Reference System (WRS-2) path/row. The 
locations of these sites are shown in Figure 7. Landsat 8 TIRS thermal images of these sites are shown in Figure 8. Note that these images are oriented in the north-south direction for cross-track MTF estimation, with the exception of the Yemen test site. This site is oriented in the east-west direction for along-track PSF/MTF estimation, but has been rotated $90^{\circ}$ in the figure for easy comparison with the other sites. As displayed in Figure 8, these images have been resampled to 30-m resolution by the Landsat Ground Processing System and obtained from the USGS EROS archive as the standard Level 1T product. Although it is somewhat difficult to see in Figure 8, the water regions at each test site are very uniform and repeatable spatially with warmer water near the shore and cooler water farther from shore. On the land side, good thermal spatial uniformity is present, but there is some variability that can affect the accurate estimation of PSF in the tail region of the curve. The number of available scenes from each site, as well as the time period of the scenes, is listed in Table 1.
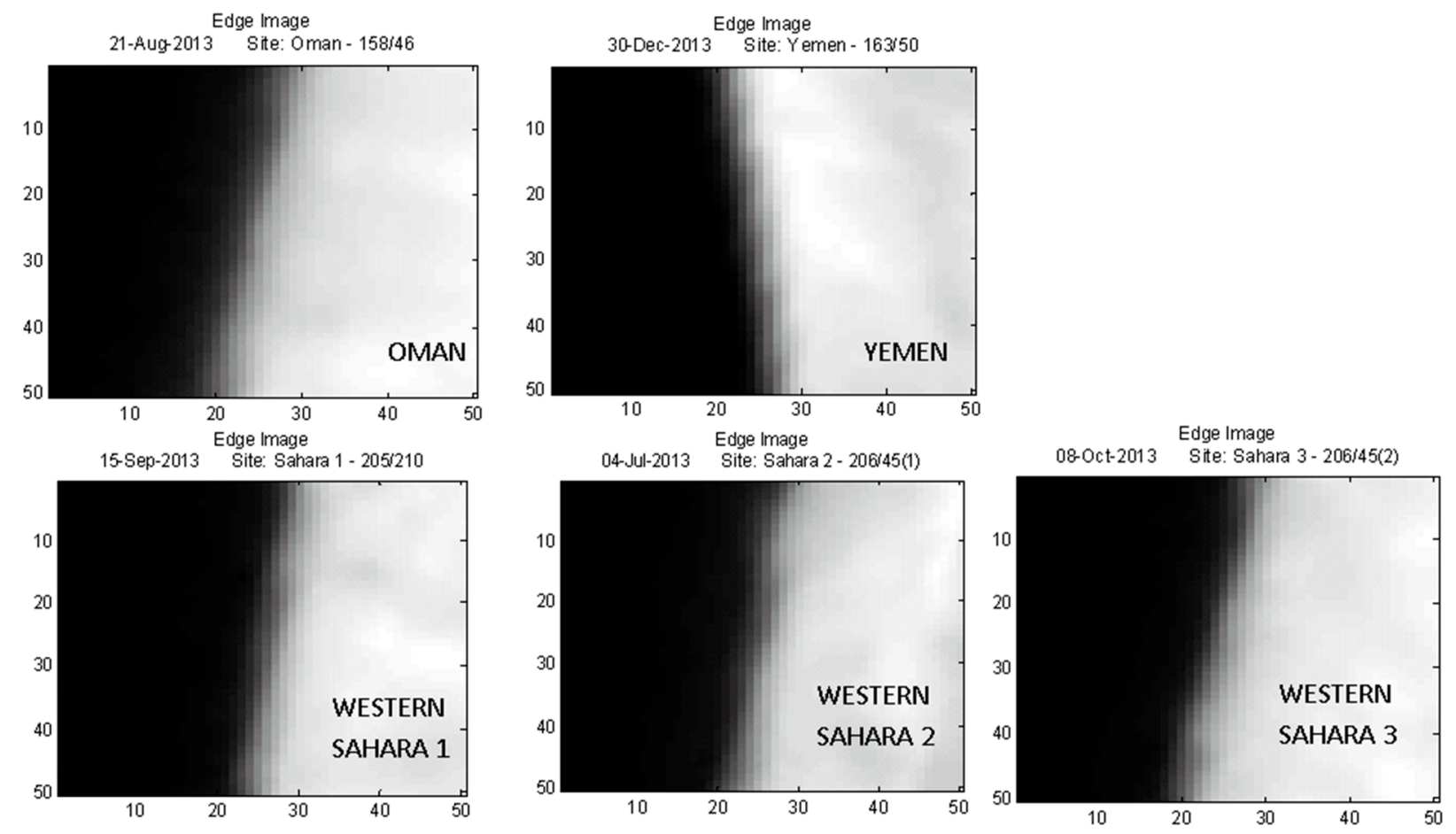

Figure 8. Landsat 8 TIRS Thermal Band images of the PSF/MTF test sites.

Table 1. List of sites, available scenes and time period.

\begin{tabular}{cccc}
\hline Site & Number of Scenes & Start Date & End Date \\
\hline Oman & 12 & 15 April 2013 & 4 May 2014 \\
Yemen & 5 & 27 October 2013 & 21 April 2014 \\
Western Sahara 1 & 8 & 11 June 2013 & 30 June 2014 \\
Western Sahara 2 & 12 & 1 May 2013 & 18 April 2014 \\
Western Sahara 3 & 12 & 1 May 2013 & 18 April 2014 \\
\hline
\end{tabular}



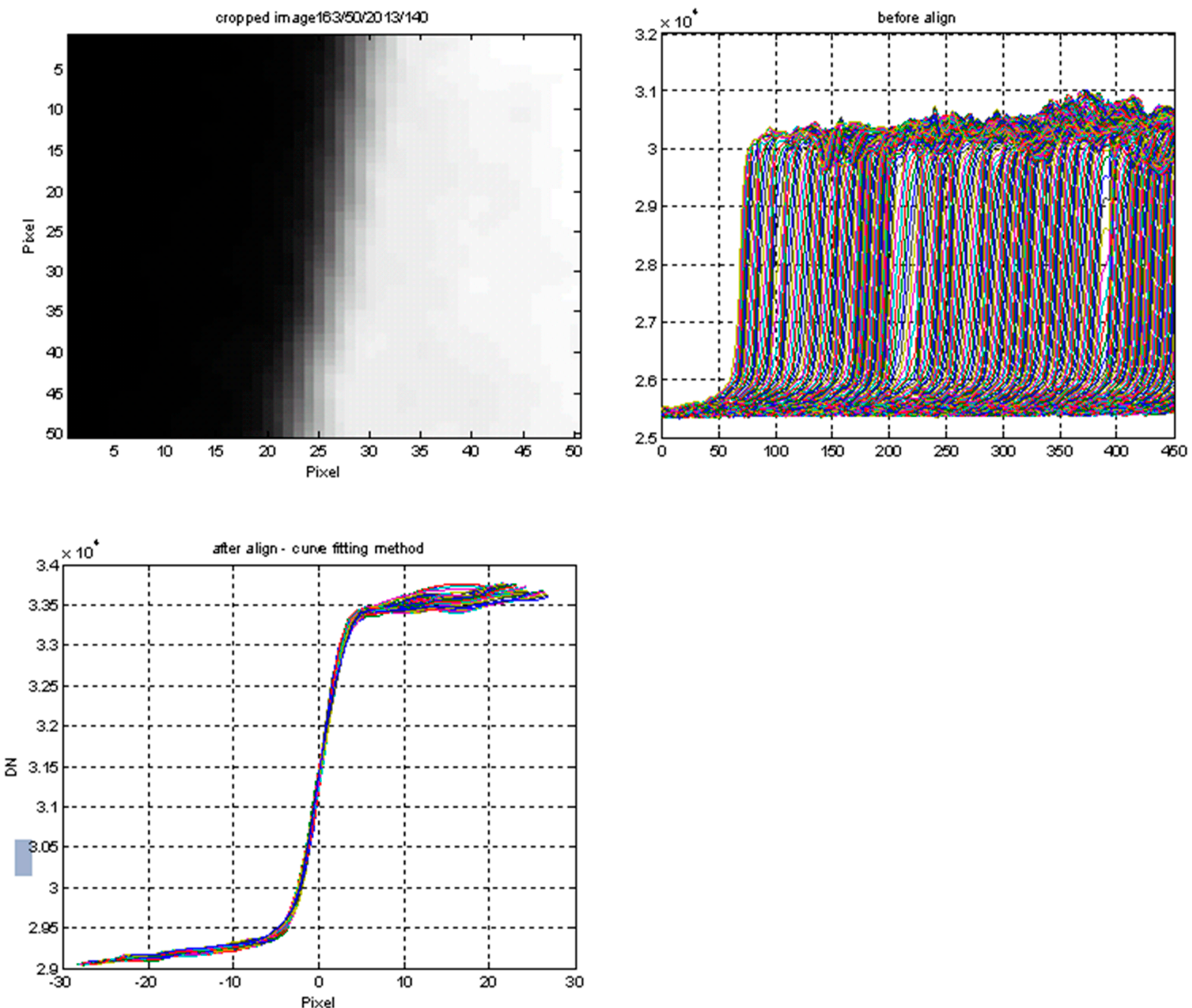

Figure 9. An illustration of the processing methodology used to obtain the PSF. (a) The original image; (b) the horizontal profile of all of the rows in the image; (c) the row profiles after alignment.

\subsubsection{Processing Steps}

The approach used to estimate the PSF/MTF of the TIRS sensor is essentially the same as is typically used with edge targets [12] and is shown visually in Figure 9. Figure 9a shows the original image, which is 50 pixels by 50 pixels in size in L1T (standard product) format with the pixel size resampled to $30 \mathrm{~m}$. Each row is extracted, and its profile is shown in Figure 9b. The first step is to identify, with sub-pixel accuracy, the exact location of the edge. There are several ways to do this, but one of the most effective is to fit a Fermi function to the data, as was done with the pre-launch analysis. While the Fermi function is not a perfect fit to the entire profile, it tends to faithfully follow the data in the steepest portion of the curve, which is where the edge is located. The equation for a modified Fermi function is:

$$
y(x)=d+\frac{b-d}{1+e^{-s(x-e)}}+g x
$$

where $d$ represents the mean value on the dark side of the edge, $b$ is the mean value on the bright side, $s$ represents the slope of the edge and $e$ is the actual edge position. Because of the nature of the edge that is 
being used, it was necessary to modify the Fermi function by adding a linear term, $g x$. This is necessary because the temperature on either side of the land/water boundary is not constant, but changes in a roughly linear fashion as the distance from the edge increases. That is, the water becomes colder farther from the edge, and the land becomes warmer farther from the edge. The modified Fermi function is fit to the data using a least squares algorithm. Once this is done, the edge profiles can be aligned based on the estimated location of the edge, as shown in Figure 9c, to obtain the edge spread function. This figure indicates the excellent alignment of the data, very uniform water gradually becoming colder farther from shore, and the amount of variability that is common on the land (bright) side of the edge. The ESF data are then normalized by subtracting out the linear portion of the modified Fermi model and scaled from zero to unity by dividing by the height of the edge $(b-d)$. An example of the resulting data is shown in Figure 10a.

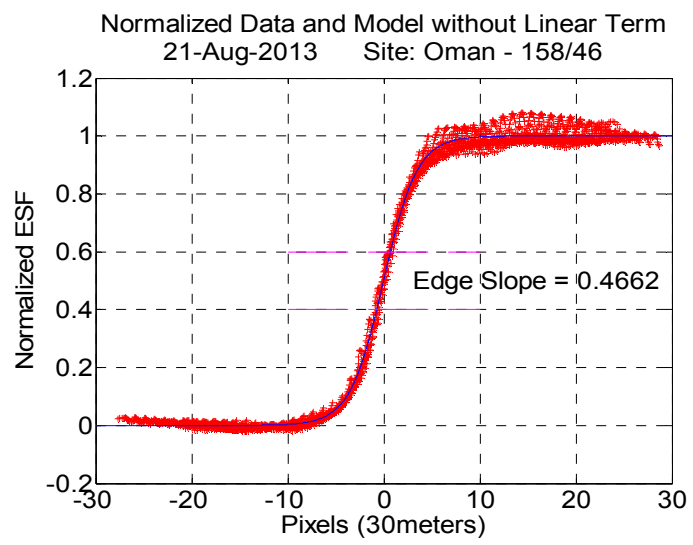

(a)

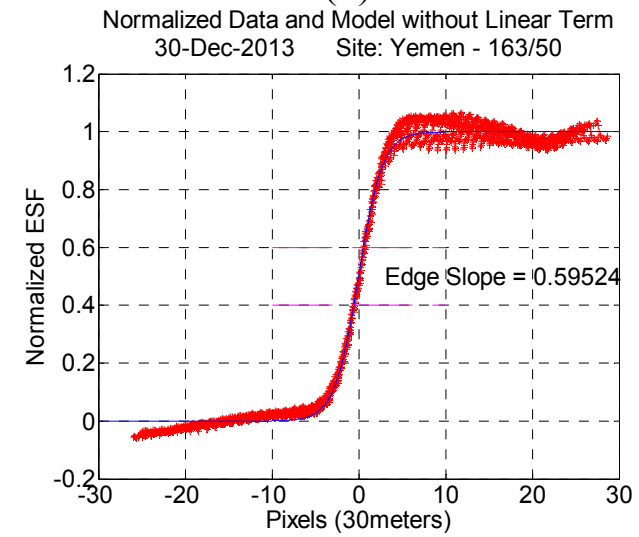

(c)

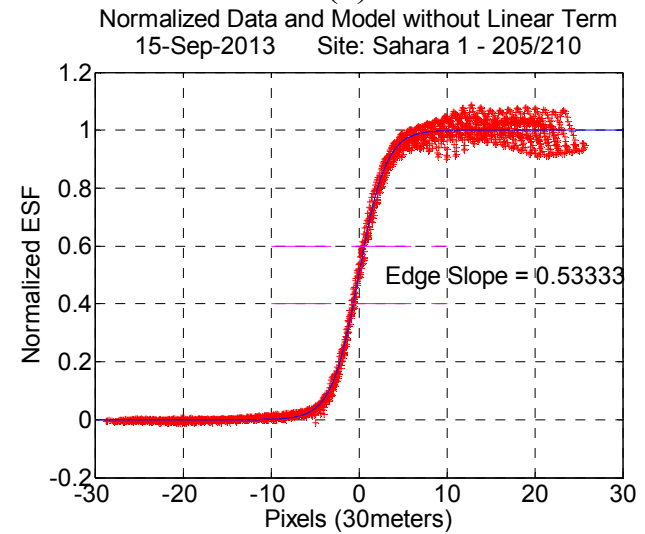

(e)

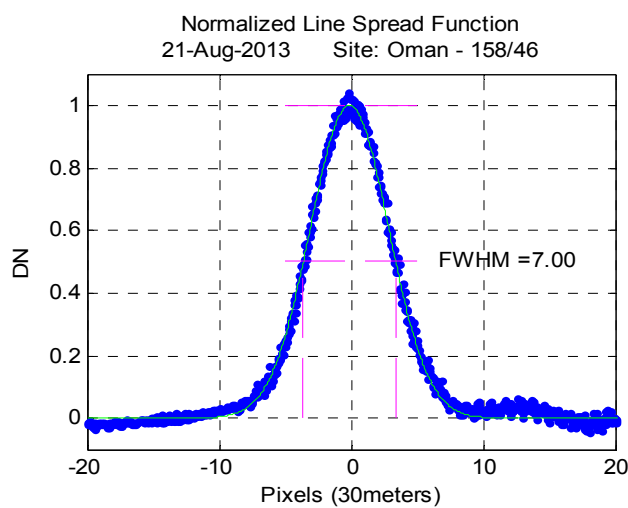

(b)

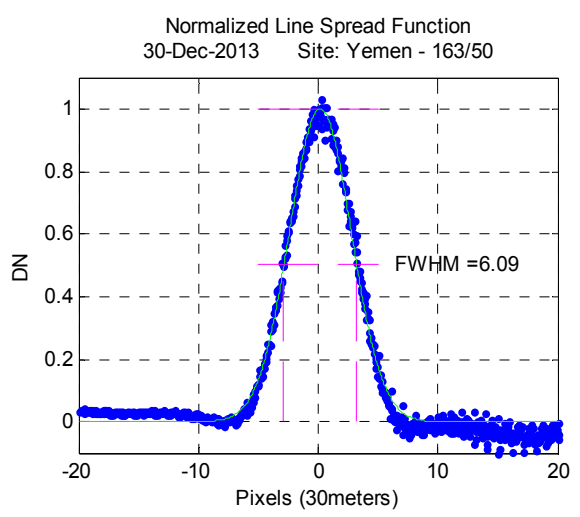

(d)

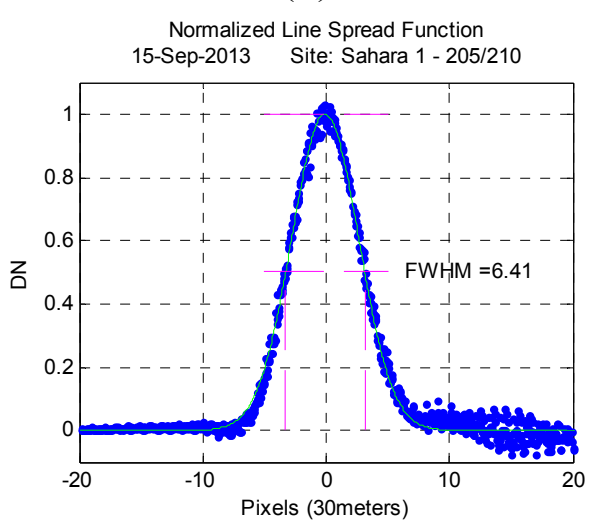

(f)

Figure 10. Cont. 


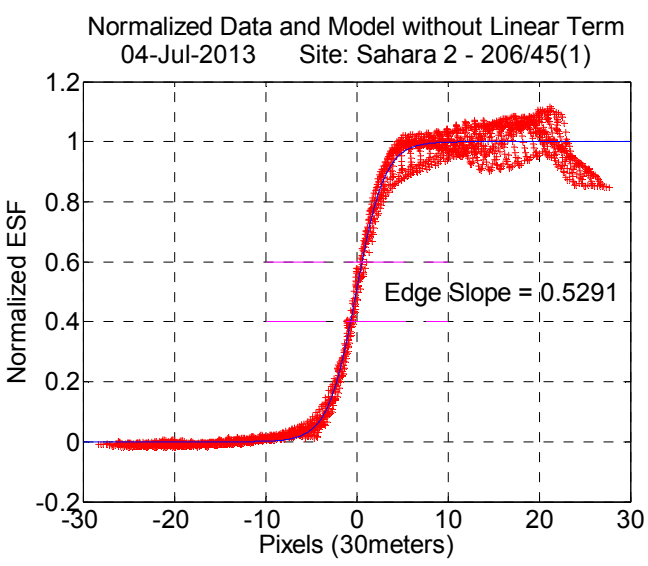

(g)

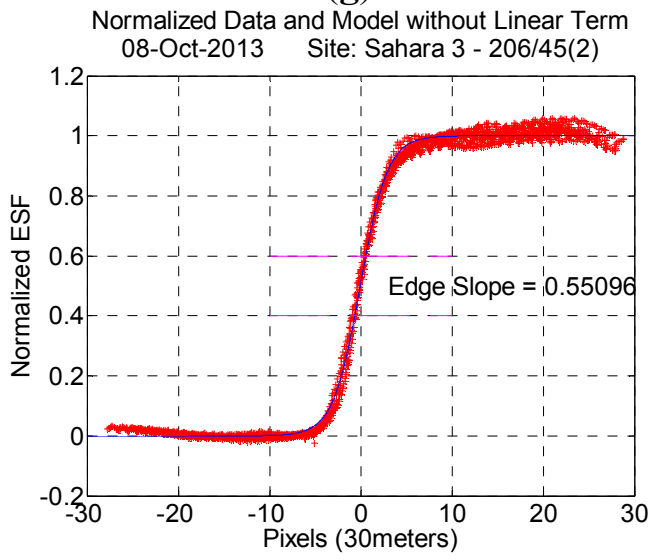

(i)

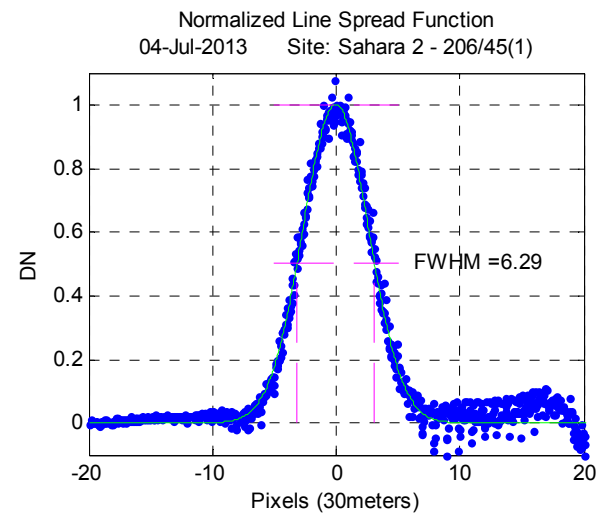

(h)

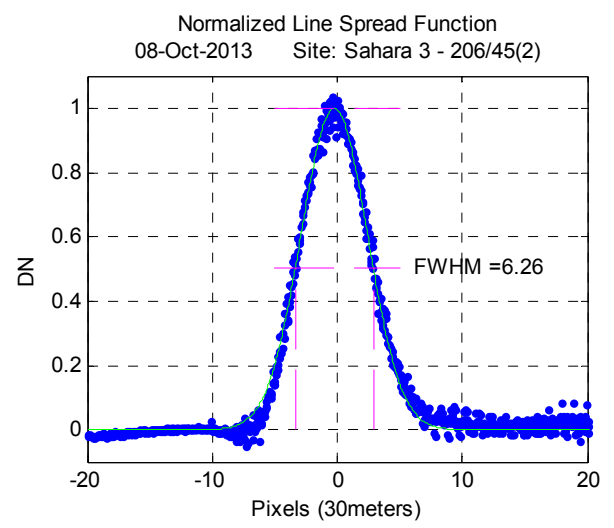

(j)

Figure 10. Edge spread function (ESF) and PSF for the five test sites: from top to bottom are (a,b) Oman, (c,d) Yemen and(e-j) Sahara 1-3. ESF data (red pluses) after normalization and the linear term removed, including the normalized Fermi model (blue line) (left). Horizontal magenta lines indicate the edge slope calculation in native pixel resolution (100 $\mathrm{m})$. The corresponding line spread function estimates (blue diamonds) with a Gaussian model (cyan line) fit, and the FWHM is overplotted on the data (right).

Once the edge profile has been established, the data were filtered to remove noise and to also provide uniform horizontal sampling. A Savitzky-Golay filter was used to accomplish this task and was modified so that it could accept data that were non-uniformly sampled [17]. In this implementation, a third order polynomial was applied to a window of data that spanned 10 pixels. The filter was incremented with a step size of 0.05 pixels to produce 20 uniformly-spaced data points for each pixel.

The last step in the processing is the differentiation of the edge spread function to obtain the PSF. This was accomplished by simply using first differences, and the result is illustrated in Figure 10b. As shown in the figure, the data indicate a fairly symmetric PSF with good signal-to-noise characteristics. For comparison purposes, a Gaussian curve is also fit to the data. 


\section{Results and Discussion}

\subsection{Pre-Launch}

A total of 26 spatial datasets were collected during TVAC testing, covering locations on each band of all three SCAs to sample the entire FOV. Several locations were subject to repeated measurements with slightly different steering mirror configurations to verify the reproducibility of the results. An example normalized edge response typical of the analysis performed is seen in Figure 6. It is evident that the analysis produces a smooth, well-sampled edge response curve with low noise. The blue horizontal dashed lines indicate the 0.4 and 0.6 edge points used to define the edge slope response. The red horizontal dashed lines denote the 0.1 and 0.9 edge points used to define the edge extent parameter. The other two sets of black dashed lines are for ripple and overshoot estimation. Table 2 lists the resulting edge slope response and edge extent for the 13 locations sampled for the $10.9-\mu \mathrm{m}$ band. For this analysis, a pixel size of $100 \mathrm{~m}$ was used, so the edge slope is in units of $1 /(100-\mathrm{m}$ pixel). The pixel column/row values in Table 2 are the edge center points, as determined in the analysis ( 0.5 edge response point in Figure 3) The cross-track edge slope values range from 0.53 to 0.60 , with a mean of $0.59(\sigma=0.02)$. The table lists the results in order of one edge of the FOV to the other cross-track, i.e., the middle of SCA-C is closest to nadir. It can be seen that there is a slight degradation in edge slope at both ends of the FOV. This is not unexpected. The along-track edge slope response displayed a wider range of values, from 0.46 to 0.56 , with a mean of $0.53(\sigma=0.03)$. As the active detector region for each SCA of a given band was only 32 pixels along-track, there are less useable pixels in the tail after the edge signal has dropped near the background levels. The edge slope along-track retrievals are therefore slightly noisier due to this, as the Fermi function fitting process is more challenging. Similar to the cross-track edge slope values, the along-track values are lower at the extreme ends of the FOV. The edge extent values listed in Table 2 have a mean of $202.8 \mathrm{~m}(\sigma=9.1 \mathrm{~m})$ cross-track and $234.0 \mathrm{~m}(\sigma=17.1 \mathrm{~m})$ along-track. Again, the edges of the FOV did not perform as well as the central portion of the FOV. Results for the 12- $\mu \mathrm{m}$ band collects are very similar, if not slightly better. The mean edge slope cross-track and along-track are $0.61(\sigma=0.01)$ and $0.63(\sigma=0.02)$, respectively. The mean edge extent cross-track and along-track are $197.6 \mathrm{~m}(\sigma=6.9 \mathrm{~m})$ and $184.3 \mathrm{~m}(\sigma=11.0 \mathrm{~m})$, respectively. Additional spatial data collects were acquired and analyzed under different environmental conditions and pre- and post-instrument vibration testing. Results for edge slope and edge extent were consistent for all datasets collected and showed no changes in spatial performance.

The main intent of the pre-launch spatial characterization was to assess instrument performance in terms of design requirements, which were stated in terms of the edge slope and edge extent. Consideration of on-orbit along-track effects, such as integration time blur, was not included in the analysis. The testing analysis results show that TIRS did not meet the spatial requirements of an edge slope $>0.7$ and an edge extent $<150 \mathrm{~m}$. However, modeling analysis using DIRSIG indicated no discernable loss of image quality and that no TIRS science objectives are compromised by not meeting the spatial response requirements [18]. A singular root cause for not meeting the challenging requirement for edge slope/extent has not been found at the time of the writing of this article. Optical modeling of the sensor, pre-launch test data and post-launch data are still being evaluated to understand the possible stray light issues better in the sensor itself, as well as from the CGSE system. 
Table 2. Pre-launch results for 10.9- $\mu \mathrm{m}$ band edge slope and edge extent.

\begin{tabular}{ccccccc}
\hline \multirow{2}{*}{ SCA } & \multirow{2}{*}{ Column } & \multirow{2}{*}{ Row } & \multicolumn{2}{c}{ Edge Slope } & \multicolumn{2}{c}{ Edge Extent (m) } \\
\cline { 4 - 7 } & & & Cross-Track & Along-Track & Cross-Track & Along-Track \\
\hline A & 602.96 & 5.65 & 0.53 & 0.46 & 230.16 & 271.80 \\
A & 262.15 & 6.33 & 0.60 & 0.51 & 198.10 & 247.40 \\
A & 23.13 & 9.91 & 0.60 & 0.52 & 200.38 & 240.34 \\
A & 19.05 & 8.40 & 0.60 & 0.54 & 197.09 & 235.02 \\
C & 21.64 & 12.01 & 0.60 & 0.56 & 199.97 & 216.49 \\
C & 24.02 & 11.35 & 0.60 & 0.55 & 201.45 & 221.29 \\
C & 315.32 & 9.28 & 0.60 & 0.55 & 195.85 & 214.96 \\
C & 601.52 & 12.36 & 0.59 & 0.56 & 204.69 & 217.83 \\
C & 602.57 & 9.55 & 0.59 & 0.55 & 204.34 & 222.73 \\
B & 601.13 & 1.03 & 0.59 & 0.53 & 198.18 & 224.76 \\
B & 599.97 & 3.48 & 0.60 & 0.52 & 197.82 & 257.29 \\
B & 263.40 & 9.82 & 0.60 & 0.51 & 197.91 & 237.75 \\
B & 23.29 & 10.38 & 0.57 & 0.48 & 210.00 & 234.55 \\
\hline
\end{tabular}

\subsection{Post-Launch}

A sample edge response function result generated from the Oman test site is shown in first row of plots in Figure 10. In this figure, the extent of the data was limited to \pm 30 pixels. The data, shown by red plus symbols, have been normalized as described in the previous section. Thus, they extend from nominally zero to one. Removal of the linear term in the modified Fermi model has resulted in fairly flat data on either side of the edge. However, it is also observed that there is significantly more noise on the warm/land side of the edge. Although additional noise will always be present on the land side of the boundary, it has little effect on the values obtained for edge slope and FWHM. This particular dataset, as well as all of the others in Figure 10, is drawn from those dates that produced edge slope and FWHM values that are close to the mean for each test site.

A signal-to-noise ratio (SNR) was calculated for each of the edge spread functions that was generated [19]. Values for the Oman test site ranged from 30 to 60. Past experience suggests that SNR $\geq 100$ is optimal for accurate PSF/MTF estimation, but that consistent estimates can be obtained from an SNR of 50 or greater [19]. The average SNR for the Oman site was 43.

Figure 10b shows the corresponding PSF obtained for the same date from the Oman test site. These results indicate that the TIRS has a fairly symmetric PSF. As expected, the land side of the PSF shows more variability than the water side. The FWHM value for this site was 7.00 pixels based on the L1T product that has been resampled to a pixel ground sample distance (GSD) of 30 meters. In the native resolution of the TIRS satellite $(\mathrm{GSD}=100 \mathrm{~m})$, the FWHM becomes 210 meters. For the Oman site, the average FWHM over all test images was $212.1 \mathrm{~m}$ with an uncertainty of $7.5 \mathrm{~m}(\mathrm{k}=1)$.

Figure 10 shows representative ESF and PSF estimates from all five test sites. Quick visual comparisons of the data can be made, suggesting qualitative advantages of one site over another. For example, the ESF from the test site Sahara 2 shows the largest amount of variability over the land region, while the Sahara 3 test site ESF shows the least amount. Conversely, on the water side of the edge, the Yemen example shows some residual decrease in temperature, which suggests that the linear 
portion of the modified Fermi model did not accurately represent the variability in the data. All of the PSF estimates show more noise on the land side of the edge and also suggest good symmetry. Noise levels at the base of the PSF curves prevent an appraisal of the presence of a slight amount of undershoot. On all example plots in Figure 10, the edge slope is given for the ESF, and the corresponding FWHM is given for the PSF estimate.

Summary data for all test sites are shown in Table 3. The Oman test site produced the smallest edge slope and the largest FWHM values and exhibits the least consistency in its estimates of FWHM. The other test sites, Yemen and the three Western Sahara sites, produced very consistent estimates (as indicated by low standard deviations) of FWHM, indicating consistent spatial quality performance for the TIRS sensor. The Western Sahara sites consistently produced very similar edge slope values. Edge slope estimates from all test sites exhibited very similar levels of uncertainty, as expressed by the standard deviation of the mean value (about \pm 0.03 ), with Western Sahara 3 much lower at 0.01. With respect to FWHM, the Western Sahara sites consistently produced the same value with a very low uncertainty of $2 \%$ (standard deviation divided by the mean) or less. It is surprising that the Yemen site produced the largest edge slope and smallest FWHM, because the orientation of the edge there is in the along-track direction and would have an additional blurring contribution due to the orbital motion of the sensor. Lastly, it is noted that SNR may not be a consistent indicator of PSF/MTF estimation performance. The lowest ranking sites for SNR are the Sahara 1 and 2 sites, yet they produce edge slope and FWHM estimates that are very similar to what was produced by the Sahara 3 site, which has much higher SNR. The highest ranking site for SNR is the Yemen site, which produces edge slope and FWHM estimates that are higher than Sahara 3, yet the average SNR values for the two sites are quite similar. However, the guideline of SNR $>50$ still seems to hold, in that Yemen and Sahara 3 produce the best overall estimates among all of the sites.

Table 3. Summary edge slope and FWHM data for all test sites.

\begin{tabular}{cccccccccc}
\hline Sites & Rotation & Path/Row & SNR & Edge Slope & SD & $\begin{array}{c}\text { FWHM } \\
\mathbf{( 3 0} \mathbf{~ m ~ P i x e l )}\end{array}$ & SD & $\begin{array}{c}\text { FWHM } \\
\text { (Meters) }\end{array}$ & SD \\
\hline Oman & $180^{\circ}$ & $158 / 46$ & 43 & 0.469 & 0.027 & 7.07 & 0.25 & 212.1 & 7.5 \\
\hline Yemen & $90^{\circ}$ & $163 / 50$ & 57 & 0.577 & 0.029 & 6.11 & 0.07 & 183.3 & 2.1 \\
\hline Western Sahara 1 & $0^{\circ}$ & $205 / 42$ & 37 & 0.528 & 0.030 & 6.41 & 0.11 & 192.6 & 3.3 \\
\hline Western Sahara 2 & $0^{\circ}$ & $206 / 45$ & 34 & 0.537 & 0.031 & 6.22 & 0.18 & 186.9 & 3.0 \\
\hline Western Sahara 3 & $0^{\circ}$ & $206 / 45$ & 54 & 0.545 & 0.014 & 6.37 & 0.13 & 191.1 & 3.9 \\
\hline
\end{tabular}

Estimates obtained at the Yemen and Sahara 3 sites are likely the most accurate and exhibit the best precision among the three sites. Since these two sites lie in the along-track and across-track directions, they represent a complete set of sites for monitoring the PSF/MTF of the TIRS instrument. Secondly, all uncertainties with this measurement technique should tend to bias the spatial quality of the sensor to a value that is poorer than the true value. This assertion is driven primarily by the nature of the test sites. In all cases, the physical attributes of the test site are such that there exists some type of transition region from cooler to warmer temperatures that will necessarily increase the width of the ESF and decrease the spatial quality estimate. Other target features, such as the approximately linearly changing temperature far from the edge and the less than ideal SNR will also tend to decrease the edge slope or increase the 
FWHM. Lastly, the data used for this study are the standard L1T product produced at USGS EROS, and the resampling that is done for geometric registration will also cause a slight reduction in spatial quality. Thus, it is likely that the true TIRS edge slope and FWHM may be slightly better than what these estimates suggest.

\section{Conclusions}

Knowledge of the spatial quality of a remote sensing instrument is an important aspect in interpreting the on-orbit operational sensor images. The TIRS instrument underwent thorough TVAC testing during pre-launch. Assessing spatial quality was part of this testing and was focused on determining whether or not TIRS met the instrument design requirements. A methodology using the CGSE circular target was developed and used to derive the relative edge response of TIRS. It was found that TIRS did not meet the design requirements in terms of edge slope and edge extent. However, subsequent modelling analysis determined that there was no discernable loss of image quality and that no TIRS science objectives are compromised by not meeting the spatial response requirements. Post-launch, a method of estimating the spatial quality of on-orbit thermal sensors using naturally occurring targets has been developed. It is based largely on previous techniques using slant edge targets. The new contribution includes the use of naturally occurring shorelines that have large differences in land and water temperature as edge targets. These types of shorelines naturally occur in desert regions and can be found with the proper orientation, such that an oversampled edge spread function can be generated. By modifying the approximating function to account for changes in temperature as a function of distance from the edge, consistent estimates of the spatial quality of the sensor can be obtained. However, the precision and, likely, accuracy of the method is a significant function of the quality of the target being used. It is difficult to directly compare the edge slopes determined from pre- and post-launch, particularly since the postlaunch analysis was performed on the standard product produced by the Landsat Ground Processing System, but they do indicate that there is no significant change in spatial quality before and after launch. Cross-track edge slope pre-/post-launch estimates are 0.59 and 0.55, while along-track edge slope pre-/post-launch estimates are 0.53 and 0.58 , respectively. These differences may reflect more the uncertainties in the estimation process and indicate that there is likely no degradation of the TIRS focus occurring during the launch and initial operation timeframe.

\section{Acknowledgments}

The authors would like to thank all participants in the TIRS pre-launch TVAC testing, in particular Matt Montanaro, Zelalem Tesfaye, Allen Lunsford and Jeff Love. Useful advice and input on spatial analysis was provided by Jason Choi and Jim Storey.

\section{Author Contributions}

All authors contributed to this work.

\section{Conflicts of Interest}

The authors declare no conflict of interest 


\section{References}

1. Williams, D.L.; Goward, S.N.; Arvidson, T.J. Landsat: Yesterday, today, and tomorrow. Photogram. Eng. Remote Sens. 2006, 72, 1171-1178.

2. Reuter, D.C.; Richardson, C.M.; Pellerano; F.A.; Irons, J.R.; Allen, R.G.; Anderson, M.; Jhabvala, M.D.; Lunsford, A.W.; Montanaro, M.; Smith, R.L. et al. The Thermal Infrared Sensor (TIRS) on Landsat 8: Design overview and pre-launch characterization. Remote Sens. 2015, 7, 1135-1153.

3. Jhabvala, M.; Reuter, D.; Choi, K.; Jhabvala, C.; Sundaram, C. QWIP-based thermal infrared sensor for the Landsat Data Continuity Mission. Infrared Phys. Technol. 2009, 52, 424-429.

4. Thome, K.; Markham, B.; Barker, J.; Slater, P.; Biggar, S. Radiometric calibration of Landsat, Photogram. Eng. Remote Sens. 1997, 63, 853-858.

5. Montanaro, M.; Levy, R.; Markham, B. On-Orbit radiometric performance of the Landsat 8 thermal infrared sensor. Remote Sens. 2014, 6, 11753-11769.

6. Holst, G.C. Electro-Optical Imaging System Performance; The International Society for Optical Engineering: Bellingham, DC, USA, 1995.

7. Schowengerdt, R.A.; Archwamety, C.; Wrigley, R.C. Landsat thematic mapper image-derived MTF. Photogram. Eng. Remote Sens. 1985, 51, 1395-1406.

8. Pagnutti, M.; Blonski, S.; Cramer, M.; Helder, D.; Holekamp, K.; Honkavaara, E.; Ryan, R. Targets, methods, and sites for assessing the in-flight spatial resolution of electro-optical data products. Can. J. Remote Sens. 2010, 36, 583-601.

9. Braga, A.B.; Schowengerdt, R.A; Rojas, F.; Biggar, S. Calibration of the MODIS PFM SRCA for on-orbit cross-track MTF measurement. Proc. SPIE 2000, doi:10.1117/12.494230.

10. Oh, E.; Cho, S.; Ahn, Y-H.; Park, Y.; Ryu J-H.; Kim, S-W. In-orbit optical performance assessment of geostationary ocean color imager. In Proceedings of the 2012 IEEE International Geoscience and Remote Sensing Symposium (IGARSS), Munich, Germany, 22-27 July 2012.

11. Helder, D.; Choi, T.; Rangaswamy, M. In-flight characterization of spatial quality using point spread functions. In Proceedings of the 2004 International Workshop on Radiometric and Geometric Calibration, Gulfport, MI, USA, 2-5 December 2004.

12. Choi, T. IKONOS Satellite on Orbit Modulation Transfer Function (MTF) Measurement Using Edge and Pulse Method, Master Thesis, South Dakota State University, Brookings, SD, USA, 2002.

13. Leger, D.; Duffaut, J.; Robinet, F. MTF measurements using spotlight. In Proceedings of the 1994 International Geoscience And Remote Sensing Symposium, Pasadena, CA, USA, 8-12 August 1994.

14. Bowen, H.S.; Dial. G. IKONOS calculation of MTF using stellar images. In Proceedings of the 2002 High Spatial Resolution Commercial Imagery Workshop, Reston, VA, USA, 25-27 March 2002.

15. Storey, J. Landsat 7 on-orbit modulation transfer function estimation. Proc. SPIE 2001, doi:10.1117/12.450647.

16. Thome, K.; Reuter, D.; Lunsford A.; Montanaro, M.; Wenny, B.N.; and Tesfaye, Z. Calibration plan for the thermal infrared sensor on the Landsat data continuity mission. In Proceedings of the 2010 CALCON Technical Conference, Logan, UT, USA, 23-26 August 2010.

17. Savitzky, A.; Golay, M. Smoothing and differentiation of data by simplified least squares procedures. Anal. Chem. 1964, 36, 1627-1639.

18. Gerace, A. College of Science, Rochester Institute of Technology, Personal communication, 2012. 
19. Helder, D.; Choi, J. On-orbit Modulation Transfer Function (MTF) measurement of QuickBird. In Proceedings of the 2003 High Spatial Resolution Commercial Imagery Workshop, Rseton, VA, USA, 19-21 May 2003.

(C) 2015 by the authors; licensee MDPI, Basel, Switzerland. This article is an open access article distributed under the terms and conditions of the Creative Commons Attribution license (http://creativecommons.org/licenses/by/4.0/). 